\title{
ON CONVERGENCE RATES IN ONE-SIDED LAW OF LARGE NUMBERS
}

\author{
A.I. Martikainen \\ Sankt-Petersburg University, Faculty of Mathematics and Mechanics \\ Stary Peterhof, 198904 St.-Petersburg, Russia
}

Convergence rates in two-sided law of large numbers for sums $S_{n}=X_{1}+\cdots+$ $X_{n}$ of independent identically distributed random variables $\left\{X_{k}, k \geq 1\right\}$ have been studied in detail by Baum and Katz, Brillinger, Erdős, Hsu and Robbins, Spitzer, Heyde and Rohatgi, Gafurov, Shirokova and others (see references in [9]). Necessary and sufficient conditions were obtained for a wide class of normalizing sequences and rates of decrease of probabilities.

Convergence rates in one-sided law of large numbers were investigated by Petrov [10], Petrov and Shirokova [11], Chow and Lai [4], Gafurov and Slastnikov [6] and Amosova [1-3]. But necessary and sufficient conditions are unknown with two exceptions. Petrov and Shirokova [10] derived necessary and sufficient conditions for the exponential rate of decrease of $\mathbf{P}\left(S_{n} \geq n \varepsilon\right)$. Another exception is due to Erickson [5], it related to the series $\sum_{n=1}^{\infty} \mathbf{P}\left(S_{n}>n \varepsilon\right) / n$.

All the papers mentioned above deal with centering by zeros (or by $n \mathbf{E} X_{1}$, in case the mean is finite). We shall study probabilities $\mathbf{P}\left(S_{n}-a_{n}>b_{n}\right)$ with an arbitrary centering sequence of constants $\left\{a_{n}\right\}$. The only assumption made on the sequence $\left\{a_{n}\right\}$ is that for every $\varepsilon>0$

$$
\lim \inf \mathbf{P}\left(S_{n}-a_{n}>-\varepsilon b_{n}\right)>0, \quad \liminf \mathbf{P}\left(S_{n}-a_{n}<\varepsilon b_{n}\right)>0
$$

(unless otherwise stated all limits are taken as $n \rightarrow \infty$ ). In fact, it is possible to choose $a_{n}=\operatorname{median}\left(S_{n}\right)$. If $S_{n} / b_{n} \stackrel{P}{\longrightarrow} 0$, then $a_{n}=o\left(b_{n}\right)$. Thus, in this case we can choose $a_{n}=0$. Furthermore, if $\mathbf{E} X_{1}$ is finite and $\left(S_{n}-n \mathbf{E} X_{1}\right) / b_{n} \stackrel{P}{\longrightarrow} 0$, then we can choose $a_{n}=n \mathbf{E} X_{1}$. Either of these two situations is under investigation in all the papers mentioned above (with the two exceptions indicated). This allows us to obtain most of the results mentioned above as corollaries of our results. Part of them (for the case $b_{n}=n$ ) are presented in [8] and [7].

We begin from the simplest case $b_{n}=n$. Let $L$ stand for the class of positive functions $f$, defined on the set of positive real numbers, satisfying the conditions: $f(x) \rightarrow 0$ as $x \rightarrow \infty$ and if $\left\{X_{n}\right\}$ is a sequence of independent random variables

Part of the symposium lecture; we do not touch here one-sided strong limit theorems. 
having a common symmetric distribution satisfying $n \mathbf{P}\left(X_{1}>n\right)=o(f(n))$, then $\mathbf{P}\left(S_{n}-a_{n}>n\right)=o(f(n))$.

It is well known that $f(x)=x^{t} \in L$ for every $t \leq 0$ and $f(x)=x^{t} M(x) \in L$ for the same $t$ and arbitrary non-decreasing, positive, slowly-varying (at infinity) function $M(x)$ (see [9, Chapter 9, Theorem 28 and supplements]). So, the definition of the class $L$ includes some information about two-sided convergence rates.

Theorem 1. Let $f \in L$. Then

$$
\mathbf{P}\left(S_{n}-a_{n}>\varepsilon n\right)=o(f(n)) \text { for every } \varepsilon>0
$$

if and only if

$$
\begin{gathered}
n \mathbf{P}\left(X_{1}<-n\right) \rightarrow 0, \\
n \mathbf{P}\left(X_{1}>n\right)=o(f(n)), \\
\mathbf{E} X_{1} I\left(X_{1}<0\right)>-\infty \quad \text { or } \int_{-n}^{-n / \log (1 / f(n))} x d F(x)=o(1) ;
\end{gathered}
$$

here $F(x)=\mathbf{P}\left(X_{1}<x\right)$.

Amosova [2] has already earlier derived necessary and sufficient conditions for (1) under the additional condition $\mathbf{E}\left|X_{1}\right|<\infty$.

Corollary 1. Let $f \in L$. If the conditions (2) and

$$
n \mathbf{P}\left(X_{1}<-n / \log (1 / f(n))\right)=o(1)
$$

hold, then (1) holds.

Now we will study the exponential rate of decrease of the sequence of probabilities $\mathbf{P}\left(S_{n}-a_{n}>n\right)$.

Theorem 2. The following conditions are equivalent:

(i) For every $\varepsilon>0$ there exist $\varrho \in(0,1)$ and $C>0$ such that

$$
\mathbf{P}\left(S_{n}-a_{n}>\varepsilon n\right) \leq C \varrho^{n}
$$

for every sufficiently large $n$;

(ii) The condition (3) holds for some $\varrho \in(0,1), \varepsilon>0$ and $C>0$;

(iii) $\mathbf{E}\left|X_{1}\right|<\infty, \mathbf{E} e^{t X_{1}}<\infty$ for some $t>0$. 
Earlier, Petrov and Shirokova [11] obtained an analogous criterion for $\mathbf{P}\left(S_{n}>\right.$ $\varepsilon n) \leq C \varrho^{n}$. They showed that this inequality is true for some $\varrho \in(0,1), \varepsilon>0$, $C>0$ and all $n$ sufficiently large, if and only if $\mathbf{E} e^{t X_{1}}<\infty$ for some $t>0$. It is interesting to notice that the result of Petrov and Shirokova is purely one-sided while Theorem 2 involves information about the left tail of the distribution of $X_{1}$.

Next we will study the case $b_{n}=n^{1 / s}, 0<s<2, s \neq 1$. The situation is very simple if $s<1$. A traditional although slightly improved technique may be used in proving the next theorem (which may be known).

Theorem 3. Let $0<s<1, t \leq 0 ; M(x), x>0$, be a non-increasing, positive, slowly-varying (at infinity) function and $r>s$.

The relation

$$
\mathbf{P}\left(S_{n}-a_{n}>\varepsilon n^{1 / s}\right)=o\left(n^{t} M(n)\right) \quad \text { for each } \varepsilon>0
$$

holds if and only if

$$
\begin{gathered}
n \mathbf{P}\left(X_{1}<-n^{1 / s}\right) \rightarrow 0, \\
n \mathbf{P}\left(X_{1}>n^{1 / s}\right)=o\left(n^{t} M(n)\right) .
\end{gathered}
$$

The relation

$$
\sum_{n=1}^{\infty} n^{(r / s)-2} \mathbf{P}\left(S_{n}-a_{n}>\varepsilon n^{1 / s}\right)<\infty \quad \text { for each } \varepsilon>0
$$

holds if and only if the conditions (5) and $\int_{0}^{\infty}|x|^{s} d F(x)<\infty$ are satisfied.

In our proof of the theorem we essentially use the fact that "the negative part" of $S_{n}$ (i.e., $X_{1} I\left(X_{1}<0\right)+\cdots+X_{n} I\left(X_{n}<0\right)$ ) is negligible in the full sum. However, this is not true when $s \geq 1$.

Theorem 4. Let $1<s<2, f \in L$. The relation

$$
\mathbf{P}\left(S_{n}-a_{n}>\varepsilon n^{1 / s}\right)=o(f(n)) \quad \text { for each } \varepsilon>0
$$

holds, if and only if the conditions (5),

$$
n \mathbf{P}\left(X_{1}>n^{1 / s}\right)=o(f(n))
$$

and

or

$$
\int_{-\infty}^{0} x^{2} d F(x)<\infty
$$

$$
D\left(n^{1 / s} / \log (1 / f(n))=o\left(n^{(1 / s)-1}\right)\right.
$$

are satisfied. Here $D(x)=\int_{-x}^{0} y^{2} d F(y)+\int_{-\infty}^{-x} y d F(y)$.

Note that condition (5) implies $D(x)<\infty$. Thus, $D(x)$ is finite in Theorem 4. 


\section{References}

[1] Amosova, N.N.: Rate of convergence in the one-sided law of large numbers. - Lith. Math. J. 16, 1977, 313-319 (English translation of Litovsk. Mat. Sb. 16, 1966, 5-12).

[2] Amosova, N.N.: On the problem of rates of convergence in one-sided law of large numbers. - Izv. Vyssh. Vchebn. Zaved. Mat. 10, 1978, 3-6 (Russian).

[3] Amosova, N.N.: On probabilities of one-sided deviations of sums of independent random variables. - Math. Notes 24, 1979, 570-575 (English translation of Mat. Zametki 24, 1978, 123-132).

[4] Chow, Y.S., and T.L. LAI: Some one-sided theorems on the tail distribution of sample sums with applications to the last time and largest excess of boundary crossings. Trans. Amer. Math. Soc. 208, 1975, 51-72.

[5] Erickson, K.B.: The strong law of large numbers when the mean is undefined. - Trans. Amer. Math. Soc. 185, 1973, 371-381.

[6] Gafurov, M.V., and A.D. Slastnikov: Some problems of the exit of a random walk beyond a curvilinear boundary and large deviations. - Theory Probab. Appl. 32, 1987, 299-321 (English translation of Teor. Veroyatnost. i Primenen. 32, 1987, 327-348).

[7] Martikainen, A.I.: One-sided variants of the law of large numbers, strong law and rates of convergence. - Proceedings of the 16th All-Union School on Probability Theory and Mathematical Statistics, held in Bakuriani on February 26-March 5, 1982; Metsniereba, Tbilisi, 1982, 45-61.

[8] Martikainen, A.I.: On the probability of the deviation of the sample mean from its median. - Zap. Nauchn. Semin. Leningr. Otd. Mat. Inst. Steklova 130, 1983, 130-136 (Russian).

[9] Petrov, V.V.: Sums of independent random variables. - Springer-Verlag, BerlinHeidelberg-New York, 1975.

[10] Petrov, V.V.: One-sided strong law of large numbers for ruled sums. - Vestnik Leningrad Univ. Math. 7, 1979, 164-168 (English translation of Vestnik Leningrad Univ. Mat. Mekh. Astronom. 7, 1974, 55-59.

[11] Petrov, V.V., and I.V. Shirokova: On exponential rate of convergence in the law of large numbers. - Vestnik Leningrad Univ. Mat. Mekh. Astronom. 7, 1973, 155-157 (Russian). 